\title{
BMJ Open Feasibility of a ballet-inspired low- impact at-home workout programme for adults with stroke: a mixed-methods exploratory study protocol
}

\author{
Suzanne Hoi Shan Lo (D) , ${ }^{1}$ Janita Pak Chun Chau, ${ }^{1}$ Kai Chow Choi, ${ }^{1}$ Jonas Yeung, ${ }^{2}$ \\ Siu Hung Li, ${ }^{3}$ Marika Demers ${ }^{4}$
}

To cite: Lo SHS, Chau JPC, Choi KC, et al. Feasibility of a ballet-inspired low-impact at-home workout programme for adults with stroke: a mixed-methods exploratory study protocol. BMJ Open 2021;11:e045064. doi:10.1136/ bmjopen-2020-045064

- Prepublication history for this paper is available online. To view these files, please visit the journal online (http://dx.doi. org/10.1136/bmjopen-2020045064).

Received 21 September 2020 Revised 08 March 2021 Accepted 16 March 2021

D) Check for updates

(C) Author(s) (or their employer(s)) 2021. Re-use permitted under CC BY-NC. No commercial re-use. See rights and permissions. Published by BMJ.

${ }^{1}$ The Nethersole School of Nursing, Faculty of Medicine, The Chinese University of Hong Kong, Shatin, Hong Kong

${ }^{2}$ Alice Ho Miu Ling Nethersole Hospital, NT, Hong Kong ${ }^{3}$ North District Hospital, NT, Hong Kong

${ }^{4}$ University of Southern California, Los Angeles, California, USA

Correspondence to Dr Suzanne Hoi Shan Lo; suzannelo@cuhk.edu.hk

\section{ABSTRACT}

Introduction Balancing problems are prominent in stroke survivors with unilateral paresis. Recent evidence supports that dance interventions are associated with significant improvements in gait, stability and walking endurance in people with neurological conditions. The aim of this study is to explore the feasibility of a novel ballet-inspired athome workout programme (Footprints to Better Balance (FBB)) for stroke survivors.

Methods and analysis A mixed-methods exploratory study incorporating a randomised controlled trial and qualitative evaluation will be conducted. We will recruit 40 adults with a first-ever ischaemic or haemorrhagic stroke and mild-moderate lower limb paresis from two acute stroke units. The intervention group will receive usual care plus FBB, an 8-week home-based programme with ballet-inspired workouts underpinned by Bandura's principles of self-efficacy and outcome expectation. FBB will be delivered by trained lay and peer volunteers, with the support of volunteer healthcare professionals. Multiple data will be collected: Recruitment rate, adherence to $\mathrm{FBB}$, semi-structured interviews and questionnaires on outcomes (balance, gait and memory) assessed at baseline and immediately post-intervention. The generalised estimating equations model will be used to compare differential changes on outcomes across time points between the two arms. Qualitative data will be coded and grouped to form themes and subthemes. Ethics and dissemination Ethical approval from the Joint Chinese University of Hong Kong-New Territories East Cluster Clinical Research Ethics Committee has been obtained. All eligible participants will provide written informed consent. Study results will be disseminated via publications in peer-reviewed journals and presentations at international conferences.

Trial registration number NCT04460794

\section{INTRODUCTION}

Stroke is ranked as the second leading cause of global deaths and a major cause of disability. ${ }^{1}$ Over $65 \%$ of stroke survivors have hemiparesis, considerably affecting their daily life and social functions. ${ }^{2}$ Substantial evidence shows that people with hemiparesis have significantly higher risks of falls, depression
Strengths and limitations of this study

- This study will establish the feasibility of a novel ballet-inspired low-impact at-home workout programme for community-dwelling stroke survivors with lower limb paresis, featuring the adoption of ballet-inspired workouts, mobilisation of community resources for capacity building and the usage of theory-driven strategies to enhance survivors' selfefficacy and outcome expectations in performing the workouts at home.

- It will be the first study of its kind to assess the feasibility and preliminary effects of a ballet-inspired at-home intervention for Chinese stroke survivors; cross-cultural applicability can be examined.

- Due to the nature of the intervention, only research assistants who will conduct recruitment, baseline and follow-up assessments will be blinded to the participants' group allocations, while it is not possible for participants and the persons who will deliver the intervention.

and stroke recurrence. Their disability is associated with increased burden on caregivers and healthcare resource utilisation. ${ }^{3}$

Balancing problems are prominent in stroke survivors with unilateral paresis. They exhibit imbalanced body alignment and gait deviations such as extension with plantar flexion of foot on the affected side, decreased walking speed and shorter stride length. ${ }^{45}$ These changes impair their postural control and functional mobility such as walking. Participation in balancing and muscle strengthening training is therefore very important. However, as over $70 \%$ of stroke survivors also develop verbal, visual or informational memory loss, their executive and social functions are impaired. It causes them to have difficulties in memorising exercise steps, and hence hinders their participation and the effectiveness of recovery training. ${ }^{6}$ 
Contemporary evidence-based guidelines recommend early discharge from hospital to enhance stroke survivors' reintegration to society. ${ }^{7}$ Hospital-based training often ends after survivors have attained a certain level of physical functions. A critical condition to sustain physical gains is the survivors' ability and willingness to continue their rehabilitation after discharge. ${ }^{7}$ Effective interventions to address their physical and cognitive needs are therefore necessary to support chronic recovery.

Dance is a combination of physical movements and musical beats. A systematic review of nine studies reports that dance interventions are associated with significant improvements in gait, stability and walking endurance in people with neurological conditions including stroke. ${ }^{8}$ Another review suggests that dance interventions offer a new framework for neurorehabilitation. ${ }^{9}$ Dance engages a person in both physical and cognitive stimulation. Repeated exercises in music and mental rehearsal of dance steps enhance ease to memorise and execute the planned sequences of movements. Simultaneous coordination of physical and cognitive activities enables dance interventions to take advantage of neuroplastic properties of the brain and bring about synergistic physical and cognitive benefits. ${ }^{89}$ The pleasurable experience and social engagement in dance interventions outweighs exercise alone as they increase adherence to interventions. ${ }^{9}$

Recent evidence supports the feasibility of dance interventions for stroke survivors. A pre-test and post-test study of 20 survivors found a 10-week dance intervention (two 60-minute classes per week) held in community settings was potentially beneficial in improving balance. The classes featured dance movements of ballet, contemporary, jazz, folk and ballroom. ${ }^{10}$ Another pre-test and posttest study of nine survivors reported that a 45-minute biweekly dance intervention integrating jazz dance and merengue offered in a rehabilitation setting improved their balance. ${ }^{11}$

Underpinning a complex intervention with a theoretical framework is integral to enable better understanding of the mechanism of changes in outcomes. ${ }^{12}$ A systematic review suggests that Bandura's construct of self-efficacy is the most commonly used theoretical premise underpinning stroke self-management programmes. ${ }^{13}$ A stroke self-management programme underpinned by Bandura's constructs of self-efficacy and outcome expectation was associated with significant improvements in satisfaction with performance of self-management behaviours and quality of life. ${ }^{14}$

There are some gaps identified in the literature. First, dance interventions examined in previous studies were not underpinned by theoretical frameworks and thus limited the understanding of mechanisms of change in outcomes. Second, only one study was conducted in community settings and it required participants to have access to a community centre to receive the dance intervention. ${ }^{10}$ Alternative means to remove physical barriers and reach more survivors would be of greater benefit. Third, current evidence showed that dance interventions for stroke survivors were all delivered by dance instructors and/or health professionals. ${ }^{10}{ }^{11}$ It is worthwhile to explore alternative approaches that can mobilise community resources more effectively and build community capacity in health promotion. Fourth, there is no study reporting the effects of dance interventions on Chinese stroke survivors.

\section{AIMS AND OBJECTIVES}

We aim to establish the feasibility of a novel ballet-inspired low-impact at-home workout programme (Footprints to Better Balance (FBB)) by comparing FBB to a control group and preliminarily estimating its effects on stroke survivors' gait, balance and memory for planning a future full-scale randomised controlled trial (RCT).

Since this is an exploratory feasibility trial, there will be no hypothesis.

Objectives are to:

1. Evaluate the recruitment rate of participants.

2. Identify the participants' attendance and adverse events during FBB.

3. Explore the facilitators, barriers and contextual factors that may influence the implementation of FBB.

4. Test the acceptability of data collection procedures.

5. Assess the preliminary effects of FBB on the participants' balance, gait and memory.

\section{METHODS AND DESIGN \\ Study design}

This is a mixed-methods exploratory study which incorporates a parallel-arm, assessor-blind RCT and qualitative evaluation.

\section{Settings}

Participants will be recruited from the acute stroke units (ASUs) of two acute public hospitals in Hong Kong. The novel FBB will be conducted face-to-face at the participants' home and followed up by phone or internet media. All baseline and post-intervention assessments will be conducted in a university laboratory.

\section{Participants}

Participants will be included if they are/have: (1) 18 years old or above, (2) clinically diagnosed with a first-ever ischaemic or haemorrhagic stroke, (3) living at home, (4) mild-moderate lower limb paresis with a modified functional ambulation classification (MFAC) of III (dependent walker) or above, (5) a Montreal Cognitive Assessment (MoCA) score $>20$, (6) able to follow threestep directions, (7) able to communicate in Cantonese and read traditional Chinese and (8) given written consent to participate in the study.

Survivors will be excluded if they are/have: (1) diagnosed with transient ischaemic attack, subdural or epidural haemorrhage, (2) cerebrovascular event(s) due to tumours or head trauma, (3) pre-existing neurological, cardiovascular or orthopaedic condition that contradict 


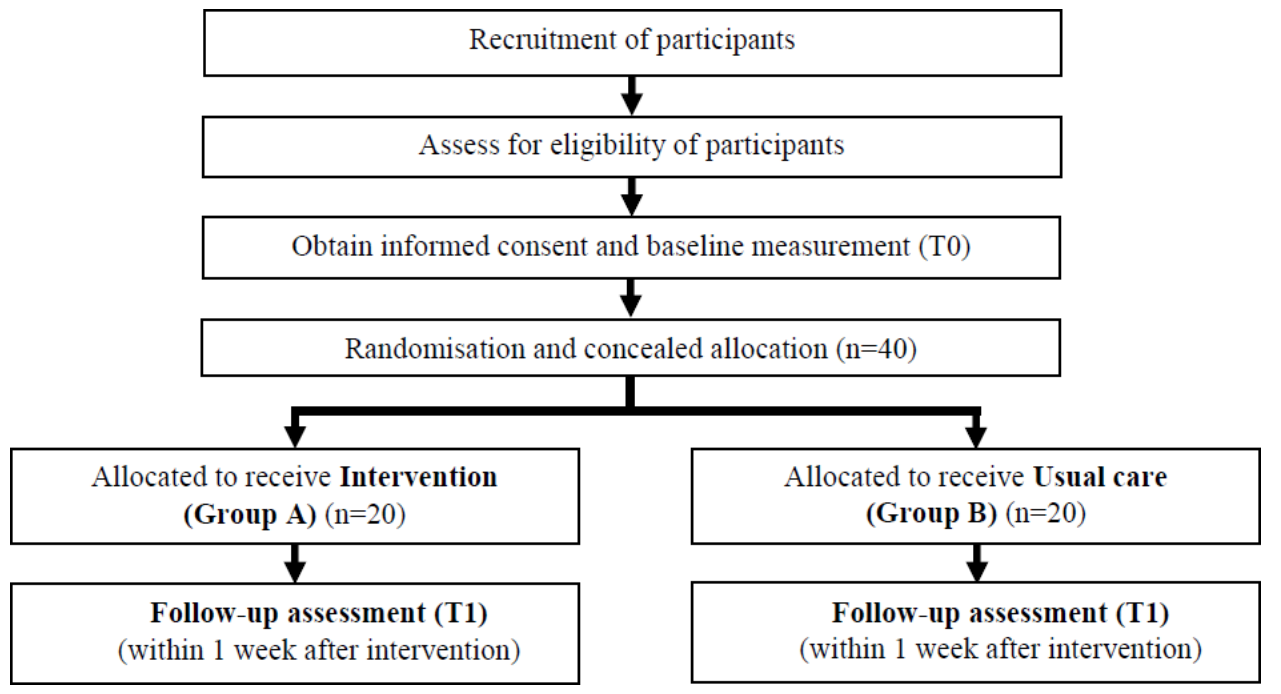

Figure 1 Flow of participants in the study.

dancing such as shoulder dislocation, myocardial infarction, seizures or acute illness, (4) mental condition such as depression, schizophrenia or personality disorder, (5) incomprehensible speech or (6) severe hearing and/or visual disturbance.

\section{Sample size calculation}

As an exploratory trial, we will recruit a total of 30 participants (15 per arm). This sample size meets the rule of thumb for sample size requirement in pilot studies. ${ }^{15}$ Allowing for a potential attrition rate of $25 \%,{ }^{13} 14$ a total of 40 eligible participants (20 per arm) will be recruited.

\section{Randomisation}

Participants will be randomly assigned at 1:1 ratio to an intervention (I) or a control (C) group after consenting and baseline assessment (see figure 1). Block randomisation (blocks of 10) will be used. An independent individual will generate a computer-generated random sequence of grouping identifiers (I or C). According to the sequence, the individual will place a grouping identifier into the opaque, identical, sealed and sequentially numbered envelopes. An independent mediator, who is not involved in recruitment, assessment or delivery of FBB, will store these envelopes in an undisclosed location, open the envelopes sequentially according to the participants' time of enrolment, record and inform the principal investigator about the participants' group allocations.

\section{Blinding}

Research assistants, who will conduct recruitment, baseline and follow-up assessments and data entry, will have no knowledge of the participants' group allocations. However, blinding is not possible for the participants and the persons delivering FBB due to the nature of the intervention. The research assistant, who will conduct qualitative evaluation with participants in the intervention group, will know the group allocation.

\section{Intervention}

Participants randomly allocated to the intervention group will receive FBB in addition to usual care. FBB is an 8-week home-based programme aimed at improving stroke survivors' balance, gait and memory. FBB was developed by the multidisciplinary healthcare team of the project in partnership with a ballet dance instructor and four stroke survivors (three women and one man, age 39-65 years, stroke duration 2-6 years). We chose ballet in lieu of other dance styles because it places emphasis on priori mastery of low-impact workouts to maintain proper body alignment, build core and lower extremity strengths and flexibility, before moving on to more complicated ballet movements. These workouts are particularly helpful for stroke survivors in correcting their balance and gait problems. Furthermore, classical ballet training emphasises motor learning for smooth performance of movements. ${ }^{16}$ When put in practice for rehabilitation training, we also emphasised to survivors on rehearsal of body movements mentally before putting the movements into actions. It mirrors mental imagery to promote motor relearning and to enhance brain plasticity and cognitive functions. ${ }^{16}{ }^{17}$ Musical beats are also integrated in ballet training, requiring coordination of both cognitive and physical activities to move the body according to the planned sequence and time. With repeated and longer duration of practice, performing ballet-inspired movements also improves cardiorespiratory fitness. The movements can be practiced alone, with partners or in groups to facilitate social engagement.

Bandura's constructs of self-efficacy and outcome expectation ${ }^{18}$ underpin the design and implementation of FBB. Strategies will be adopted to enhance participants' selfefficacy and outcome expectations of performing balletinspired workouts. ${ }^{1314}$

Eight carefully selected ballet-inspired workouts are integrated ${ }^{16}{ }^{17}$ : basic body positions, trunk movement, pointed toes, turn in and out, tendus (sliding and 
extending foot), plies (bending knees), eleves (lifting up on balls of feet) and coupes (shifting body weight). The workouts are aimed at enhancing participants' awareness of body parts and ability in maintaining proper body alignment and postural control. Participants will perform the workouts starting from a sitting position and progress to a standing position with or without physical support as their postural control improves. They will perform mental imagery of each workout after viewing demonstrations, and memorising the movements before performing. Each workout is designed to resemble a daily activity commonly performed by women or men.

We will integrate the workouts into a 60 -minute structured session adapted from a typical ballet class. ${ }^{4}$ To maintain an appropriate level of challenge, the difficulty of the workouts will increase progressively subject to participants' willingness and improved condition.

FBB will be delivered by trained lay and peer stroke volunteers with the support of volunteer healthcare professionals. The lay volunteers will provide home visits and virtual sessions to participants. The healthcare professionals will provide expert advice to volunteers during implementation. All volunteers will receive 4 days of structured training conducted by the principal investigator with over 10 years of ballet experience. Lay and peer stroke volunteers will be asked to complete an exit test to demonstrate the ability to deliver the FBB independently. Training completion will be determined by a satisfactory performance in the test and completion of one supervised on-site session and one virtual session.

A self-directed resource package will be developed in form of a website and guidebook for participants' convenience of access. It will contain videos to demonstrate the workouts, animated videos to illustrate the information and a suggested weekly goal-and-action plan for 8 weeks.

FBB will consist of two weekly 90-minute at-home support sessions delivered by two lay volunteers (one of them will be a stroke survivor) in weeks $1-2$, and six weekly 15-minute virtual interactions (by phone or internet media) by either lay volunteer in the remaining weeks. Participants will be asked to perform the 60-minute session two times per week during these 8 weeks. The home-based sessions will introduce participants to FBB, the resources package and safety precautions. The lay volunteers will conduct virtual sessions and discuss strategies to address challenges in performing workouts, reinforcing outcome expectations, appraising incremental progress and reinforcing participation as planned for the following weeks. They will update the healthcare professionals about the participant's progress, and consult them for advice if needed. All adverse events will be documented and reported to the clinical research ethics committee.

Strategies will be adopted to ensure safety of the participants during FBB. Participants are reminded to perform FBB each time starting from a sitting position and progressing to a standing position as their postural control improves. Family members or carers are encouraged to join FBB with participants and/or provide standby support to participants while they are doing FBB. The preparation of environment include preparing for a chair without wheels for support, adequate space and light and a phone nearby for making contacts when necessary. The breaks are mandatory to avoid overexertion.

\section{Control group}

Control participants will receive usual care including usual stroke services available to the participants, including but not limited to, medical consultations offered by hospital, rehabilitation services by community-based organisations. In addition, they will be provided with an information sheet about recommendations with pictorial demonstrations on basic stretching and leg exercises for stroke survivors.

\section{Recruitment and data collection procedures}

A research assistant will visit the ASUs regularly to screen for eligible participants. He/she will review the medical records of all patients who had a stroke admitted, and approach the potentially eligible participants and explain to them and/or their relatives the study aim, objectives, intervention and data collection procedures. Participants will be asked to sign an informed consent form and will be given a participation card indicating their recruitment into the study. Then, the research assistant will record the participants' demographic and clinical information. After the patients are discharged from the hospital, the research assistant will contact them and schedule a baseline assessment. Participants will be informed about video-taking during assessment of their balance and gait. Face-to-face focus group interviews with all participants in the intervention group and all volunteers will be conducted immediately post-intervention in a university laboratory room. All interviews will be audio-taped. Cash allowance will be provided to participants after completing each assessment and interview; and to volunteers after completing a home visit to subsidise their travel expenses in the study.

\section{Data collection}

Multiple data will be collected:

1. Recruitment: Review the research assistant's recruitment records and flow of participants in the study to calculate the participants' recruitment rate and the reasons for non-participation.

2. Characteristics of eligible and included/non-included adults with stroke: Participants' age, gender, marital status, educational level, stroke history, comorbidities, living condition and financial status will be extracted from the medical records.

3. Participant characteristics (completed vs dropout): Data such as age, gender, marital status, educational level, occupation, current financial aids received, type of housing, living condition, past and present medical history, assistive aids used, MoCA and MFAC scores will be extracted from the participants' records. 
4. Home journal: Participants will document details of their participation in FBB in the website or guidebook, including date, time, number of workouts performed, presence of dyspnoea, injuries or accidents.

5. Audio records: All home visits and virtual sessions of FBB, and volunteer training sessions will be audio recorded with the participants' and the volunteers' consent.

6. Qualitative evaluation: Focus group semi-structured exit interviews will be conducted by an independent research assistant with (1) all participants in the intervention group to elicit their experiences of participating in FBB, facilitators of and barriers to participating in FBB, perspectives on feasibility, acceptability and usefulness of FBB, changes in behaviours after FBB, impression of research experience and areas for enhancement; and (2) all volunteers to elicit their perceptions on the facilitators of and barriers to implementing FBB, perspectives on feasibility, acceptability and usefulness of FBB and observations of the participants' participation in FBB.

7. Outcomes: All participants will be assessed at baseline (T0) and at immediately post-intervention (T1) (within 1 week after the intervention).

- Balance: The 14-item Mini-Balance Evaluation Systems Test will be used. ${ }^{19}$ It measures four domains including the participants' anticipatory postural adjustments, reactive postural control, sensory orientation and dynamic gait. All items are rated on a three-level scale $(0=$ severe, $1=$ moderate, $2=$ normal). The summed total score is 0 to 28. A higher score represents better balance ability. The Cronbach alpha is $0.89-0.94 .^{19}$

- Balance confidence: The 16-item Activities-specific Balance Confidence Scale (Chinese version) ${ }^{20}$ will be adopted. The participants will rate their confidence in balance associated with performing 16 daily functional activities from $0 \%$ (absolutely no confidence) to $100 \%$ (fully confident). The summed total score is $0 \%$ to $100 \%$. A higher score denotes higher confidence. The Cronbach alpha is $0.97 .^{20}$

- Gait: The 31-item Gait Assessment and Intervention Tool (G.A.I.T.) will be used to measure the participants' gait: upper extremity and trunk movement control; trunk and lower extremity (stance phase); trunk and lower extremity (swing phase). Each item is scored from 0 (normal) to 3, with gradients of variation from normal. The total score ranges from 0 (normal gait) to 62 (greatest extent of gait deviations). G.A.I.T. demonstrates good intra-rater and inter-rater reliability. ${ }^{21}$

- Walking endurance: The 6-minute walk test (MWT) will be performed in accordance with the American Thoracic Society guidelines. ${ }^{22}$ The distance walked, the time stopped and reason(s) for stopping prematurely will be recorded. The 6MWT, 12MWT and self-paced gait speed were all significantly highly correlated $(r>0.90) .{ }^{23}$
- Memory: The 11-item Rivermead Behavioural Memory Test-Third Version (Chinese version) will be used to measure the participants' memory function for performing daily tasks. For each task, the scores range from 0 to 2 ( 0 point=error; 1 point=intermediate; 2 points=normal). The total score ranges from 0 to 254 . The higher the score, the better the memory performance. The test demonstrates high inter-rater reliability. The correlation between performance on parallel forms is $0.67-0.84 .^{24}$

\section{Data analysis}

All quantitative data will be summarised and presented using appropriate descriptive statistics. Recruitment rate will be calculated by the average of participants recruited per study venue per month. Cohen's d values will be calculated to estimate the effect sizes of the intervention on the outcome variables. All statistical analyses will be performed using IBM SPSS V.24.0. Raw audio files will be transcribed verbatim and destroyed after completing transcription. The interview transcripts and participants' home journals will be transcribed verbatim from the audio recordings by an independent research assistant and analysed thematically. Initial codes will be developed by two independent researchers (SHSL and JPCC), and grouped them to form major themes and subthemes that correspond to the study aim and objectives. Discrepancies in the major themes and subthemes will be resolved by discussion between the two researchers. The qualitative data will supplement the quantitative outcome data by identifying convergence and differences between the two data sets. ${ }^{25}$

\section{Patient and public involvement}

FBB was developed in partnership with a ballet instructor and four stroke survivors. Community-dwelling stroke survivors will be recruited to participate in the study. Adult lay and peer stroke volunteers will be recruited and trained to deliver FBB. Comments on the programme such as acceptability and usefulness, and areas of enhancement will be collected from the participants and the volunteers through semi-structured interviews. Preliminary effects of FBB will be assessed by the administration of questionnaires with the participants. The results of the study will be disseminated to the participants on request.

\section{Reporting guidelines}

Standard Protocol Items: Recommendations for Interventional Trials reporting guidelines were adhered to in this protocol. ${ }^{26}$

\section{ETHICAL CONSIDERATIONS AND DISSEMINATION}

Ethical approval has been obtained from the Joint Chinese University of Hong Kong-New Territories East Cluster Clinical Research Ethics Committee (Ref. No.: 2019.598). The research team will protect participants' rights and safety by adhering to local laws, the Declaration 
of Helsinki, institutional policies and the International Conference on Harmonization-Good Clinical Practice. All research personnel will be asked to complete the modules of Good Clinical Practice. Agreement will be made in advance with the personnel in charge of ASUs for arranging participant recruitment. All eligible participants will provide written informed consent. All questionnaires will be anonymous. All information will be kept strictly confidential. All information will be destroyed 6 years after completion of the project. Study findings will be disseminated via publications in peer-reviewed journals and presentations at international conferences.

Acknowledgements We would like to thank the stroke survivors and the dance teacher for providing their valuable suggestions in the development of the dance intervention.

Contributors SHSL and JPCC contributed to the conception and design of the study. MD, KCC, JY and SHL commented on the intervention contents. KCC was responsible for sample size calculation and statistical analyses. All authors are the applicants of the grant submission. SHSL wrote the manuscript and all authors read and approved the manuscript.

Funding This work is supported by the Health and Medical Research Fund (Grant Ref. No.: 17180261) from the Food and Health Bureau, Government of the Hong Kong Special Administrative Region.

Competing interests None declared.

Patient and public involvement Patients and/or the public were involved in the design, or conduct, or reporting, or dissemination plans of this research. Refer to the Methods section for further details.

Patient consent for publication Not required.

Provenance and peer review Not commissioned; externally peer reviewed.

Open access This is an open access article distributed in accordance with the Creative Commons Attribution Non Commercial (CC BY-NC 4.0) license, which permits others to distribute, remix, adapt, build upon this work non-commercially, and license their derivative works on different terms, provided the original work is properly cited, appropriate credit is given, any changes made indicated, and the use is non-commercial. See: http://creativecommons.org/licenses/by-nc/4.0/.

\section{ORCID iD}

Suzanne Hoi Shan Lo http://orcid.org/0000-0002-5933-9290

\section{REFERENCES}

1 Benjamin EJ, Virani SS, Callaway CW, et al. Heart disease and stroke statistics-2018 update: a report from the American heart association. Circulation 2018;137:e67-492.

2 Wist S, Clivaz J, Sattelmayer M. Muscle strengthening for hemiparesis after stroke: a meta-analysis. Ann Phys Rehabil Med 2016:59:114-24.

3 Ballester BR, Maier M, Duff A, et al. A critical time window for recovery extends beyond one-year post-stroke. J Neurophysiol 2019;122:350-7.

4 Billinger SA, Arena R, Bernhardt J, et al. Physical activity and exercise recommendations for stroke survivors: a statement for healthcare professionals from the American heart Association/ American stroke association. Stroke 2014;45:2532-53.
5 Li S, Francisco GE, Zhou P. Post-Stroke hemiplegic gait: new perspective and insights. Front Physiol 2018;9:1021.

6 Tang EYH, Price C, Stephan BCM, et al. Gaps in care for patients with memory deficits after stroke: views of healthcare providers. BMC Health Serv Res 2017;17:634.

7 Royal College of Physicians. National clinical guideline for stroke [Internet], 2016. Available: https://www.strokeaudit.org/SupportFiles/ Documents/Guidelines/2016-National-Clinical-Guideline-for-Stroke5t-(1).aspx [Accessed cited 18 March 2018].

8 Patterson KK, Wong JS, Prout EC, et al. Dance for the rehabilitation of balance and gait in adults with neurological conditions other than Parkinson's disease: a systematic review. Heliyon 2018;4:e00584.

9 Dhami P, Moreno S, DeSouza JFX. New framework for rehabilitation - fusion of cognitive and physical rehabilitation: the hope for dancing. Front Psychol 2014;5:1478.

10 Patterson KK, Wong JS, Nguyen T-U, et al. A dance program to improve gait and balance in individuals with chronic stroke: a feasibility study. Top Stroke Rehabil 2018;32:1-7.

11 Demers M, Thomas A, Wittich W, et al. Implementing a novel dance intervention in rehabilitation: perceived barriers and facilitators. Disabil Rehabil 2015;37:1066-72.

12 Craig P, Dieppe P, Macintyre S, et al. Developing and evaluating complex interventions: the new medical Research Council guidance. BMJ 2008;337:a1655.

13 Lo SHS, Chang AM, Chau JPC. Stroke self-management support improves survivors' self-efficacy and outcome expectation of selfmanagement behaviors. Stroke 2018;49:758-60.

14 Lo SHS, Chau JPC, Chang AM, et al. Coaching ongoing momentum building on stroKe rEcovery journeY ('COMBO-KEY'): a randomised controlled trial protocol. BMJ Open 2019;9:e027936.

15 Lancaster GA, Dodd S, Williamson PR. Design and analysis of pilot studies: recommendations for good practice. J Eval Clin Pract 2004:10:307-12.

16 Scheidler AM, Kinnett-Hopkins D, Learmonth YC, et al. Targeted ballet program mitigates ataxia and improves balance in females with mild-to-moderate multiple sclerosis. PLoS One 2018;13:e0205382.

17 López-Ortiz C, Egan T, Gaebler-Spira DJ. Pilot study of a targeted dance class for physical rehabilitation in children with cerebral palsy. SAGE Open Med 2016;4:205031211667092.

18 Bandura A. Self-Efficacy: the exercise of control. New York: W. H. Freeman, 1997.

19 Tsang CSL, Liao L-R, Chung RCK, et al. Psychometric properties of the Mini-Balance evaluation systems test (Mini-BESTest) in community-dwelling individuals with chronic stroke. Phys Ther 2013;93:1102-15.

20 Mak MK, Lau AL, Law FS, et al. Validation of the Chinese translated Activities-Specific balance confidence scale. Arch Phys Med Rehabil 2007;88:496-503.

21 Daly JJ, Nethery J, McCabe JP, et al. Development and testing of the gait assessment and intervention tool (G.A.I.T.): a measure of coordinated gait components. J Neurosci Methods 2009;178:334-9.

22 ATS Committee on Proficiency Standards for Clinical Pulmonary Function Laboratories. Ats statement: guidelines for the six-minute walk test. Am J Respir Crit Care Med 2002;166:111-7.

23 Dalgas U, Severinsen K, Overgaard K. Relations between 6 minute walking distance and 10 meter walking speed in patients with multiple sclerosis and stroke. Arch Phys Med Rehabil 2012;93:1167-72.

24 Fong KNK, Lee KKL, Tsang ZPY, et al. The clinical utility, reliability and validity of the Rivermead behavioural memory Test-Third edition (RBMT-3) in Hong Kong older adults with or without cognitive impairments. Neuropsychol Rehabil 2019;29:144-59.

25 Creswell JW, Fetters MD, Ivankova NV. Designing a mixed methods study in primary care. Ann Fam Med 2004;2:7-12.

26 Chan A-W, Tetzlaff JM, Gøtzsche PC, et al. Spirit 2013 explanation and elaboration: guidance for protocols of clinical trials. BMJ 2013;346:e7586. 